\title{
Aqueous heparin therapy: Intermittent $v$ continuous administration
}

\author{
NATHAN FREED, DO \\ THOMAS P. MORLEY, DO \\ STEPHEN DALY, DO \\ JAMES C. GIUDICE, DO, FCCP \\ ROBERT A. STEER, EDD
}

\begin{abstract}
The incidence of hemorrhagic and rethrombotic complications using continuous and intermittent doses of heparin were studied. Determination of reasons for any differences among these parameters and whether activated partial thromboplastin times (APTTs) were predictive of heparin-induced complications. Thirty-three patients were considered to be evaluable, and each was assigned randomly to one of four groups, using a two-factor design, as follows: either intermittent or continuous administration, with or without APTT-based adjustments in dosage. The groups demonstrated no significant mean differences. Surprisingly, there were no significant differences with respect to bleeding or rethrombosis. It generally is accepted that high-risk patients bleed more readily during heparin therapy, especially when it is administered intermittently. Results did not indicate that the method of administration was a significant factor or that the APTT was a reliable predictor.
\end{abstract}

A sulfate-containing mucopolysaccharide with a molecular weight of 16,000 , heparin is found in a wide range of body tissues. However, it is not freely circulating, and it is removed from the circulation chiefly by the reticuloendothelial system. ${ }^{1,2}$ Once heparin is absorbed by the vascular endothelium, it can be returned to the circulation. ${ }^{3}$

\section{Pharmacokinetics \\ Half-life}

According to Dr. TL Goodman, Dr. ME Todd, and Dr. EI Goldsmith, (written communication, 1985), the half-life of heparin varies from as short a duration as 30 minutes to as long as 360 minutes. The half-life was reported by Estes and Poulin ${ }^{4}$ to average 90 minutes in normal persons. Nyman and associates $^{5}$ have shown the half-life to be dosedependent (40 minutes when 3,000 units of heparin were injected intravenously, and 69 to $83 \mathrm{~min}$ utes when 10,000 units were used).

Following pulmonary embolism, the mean halflife of heparin has been reported ${ }^{6,7}$ to decrease to $50 \%$ of normal. While the cause of this decrease is not clear, some researchers have suggested an association with the increase in release of platelet factor $4,{ }^{8}$ while others found platelet factor 4 to be unrelated ${ }^{9}$ Hirsh and colleagues ${ }^{6}$ and Simon and coauthors ${ }^{7}$ noted that venous thromboses had no effect on heparin half-life.

With hepatic cirrhosis, a half-life $50 \%$ greater than normal has been observed, ${ }^{10}$ and some investigators ${ }^{11}$ have suggested an increased half-life in patients with impaired renal function. The various fluctuations in half-life illustrate some of the difficulties in deciding on a standard dosage schedule.

\section{Anticoagulant properties}

Thrombin acts upon fibrinogen and converts it to fibrin monomer, which subsequently polymerizes. By neutralizing the effects of thrombin on fibrinogen, heparin blocks fibrin formation. Besides its 
primary function as an antithrombin, heparin also acts as cofactor to a normally circulating globulin and anticoagulant, antithrombin III.

Anticoagulation is accomplished through acceleration of the normal rate at which antithrombin III neutralizes enzymatic-proteolytic effects of several serine proteases. By itself, antithrombin III provides little effective inhibition for other serine proteases, but with heparin present, inhibition by antithrombin III is nearly instantaneous. ${ }^{12}$ With heparin, antithrombin III rapidly inhibits activated factors 9,11 , and 12 , as well as plasmin. ${ }^{13}$ Heparin also plays a major role in facilitating the inhibition of thrombosis in the intrinsic clotting system, as well as in the common final pathway that leads to fibrin gel formation. Rosenberg and coworkers ${ }^{13}$ point out that heparin functions as a catalyst in this setting, by increasing the rate of the proteaseinhibitory reaction without being consumed and without altering the final products of the reaction.

\section{Heparin excretion}

The exact route of elimination of exogenously administered heparin and its in vivo kinetics were pointed out by Cees and associates. ${ }^{14}$ Through experimental administration of heparin by continuous infusion, they observed that there is a nonlinear diminution of potency with respect to time. The best explanation for this is saturable and linear removal mechanisms working in parallel. Desulfating enzymes could be the mechanism for saturable elimination, according to findings from animal and human studies. ${ }^{15}$ The second (linear) component of the model, for the removal of larger doses of heparin as intact molecules, appears to be excretion through the urinary tract. ${ }^{14}$

The disappearance of the anticoagulant activity of heparin, therefore, follows nonlinear kinetics and is best described as a combination of saturable (desulfated) and linear (intact urinary excretion) mechanisms.

\section{Previous dosage studies}

There is no consensus about the optimal dose of heparin, the frequency of administration, or the method of regulating dosage. The first convincing data favoring continuous infusion of heparin intravenously comes from Saltzman and associates. ${ }^{16}$ The 209 patients in their study were divided into three groups. The first group included 68 patients, who were given heparin every four hours; no adjustments were made for activated partial thromboplastin times (APTTs). The second group of 72 patients also received heparin every four hours, but the APTT levels were monitored and the heparin dose was adjusted accordingly. The 69 patients in the third group received continuous heparin infusion, with APTT adjustments.

Results indicated no difference among the three groups with respect to recurrent thrombotic episodes; one rethrombosis occurred in each group. The major difference was a $1 \%$ bleeding complication rate for the continuous heparin group $v$ bleeding incidences of $8 \%$ for intermittent heparin administration with APTT adjustment and $10 \%$ for intermittent heparin infusion without APTT monitoring. Less heparin was required with the continuous infusion method than with either of the two intermittent procedures.

Glazier and coauthors ${ }^{17}$ reviewed a patient population of $41 ; 20$ received continuous heparin infusion, and 21 had an intermittent dosage schedule. In the continuous infusion group, there were no major bleeding problems, while one third of the patients in the intermittent group experienced major bleeding. The mean dose of heparin administered intermittently was $1,367 \mathrm{U} / \mathrm{hr}$, compared with $1,062 \mathrm{U} / \mathrm{hr}$ for continuous administration. The mean amount of heparin received hourly by patients with major bleeding was $1,573 \mathrm{U} / \mathrm{hr}(48 \%$ higher than the mean dosage for the continuous heparin group).

In a 1981 study by Wilson and coworkers, ${ }^{18}$ patients with pulmonary embolism or deep-vein thrombosis were assigned randomly to receive either intermittent or continuous intravenous heparin therapy. These researchers found that patients with an enhanced risk of bleeding prior to the initiation of heparin therapy more often bled during intermittent administration. However, a greater incidence of thromboembolic recurrences was seen in the continuous heparin group. The authors suggested that this was related to the lower doses of heparin received by the continuous infusion group. They concluded that patients with a low risk for hemorrhagic complications prior to heparin use should be considered for intermittent administration, while those who are at high risk for bleeding should receive continuous infusion, because the risk and danger of bleeding outweighed the risk of rethrombosis. This was a compromise position. Continuous heparin infusion has now become almost standardized treatment, and no further prospective studies comparing bleeding and recurrent thrombosis rates for low-risk patients receiving continuous or intermittent heparin have been reported.

Hattersley and associates ${ }^{19}$ studied 134 patients with deep venous thrombosis or pulmonary thromboembolism. It was their contention that tests mea- 
uring the adequacy of anticoagulation, such as the APTT tests, generally were not good estimators of hemorrhagic risk. The activated coagulation time (ACT) parameter developed by Hattersley was used in this study, as follows: An intravenous bolus of about $50 \mathrm{U} / \mathrm{kg}$ of aqueous heparin was followed by 15 to $25 \mathrm{U} / \mathrm{kg} / \mathrm{hr}$ in continuous infusion; the continuous dosage was modified when the ACT reached a level between 150 to $190 \mathrm{sec}-$ onds. Oral Coumadin therapy was begun two or three days after the ACT came into target range. When the prothrombin time reached $21 / 2$ times the control value, heparin use was discontinued.

No heparin failure was encountered in these 134 patients, and all patients whose ACT level was within the target range responded promptly. Unexplained bleeding occurred in $50 \%$ of patients whose ACT level was more than 190 seconds; two of these patients experienced major bleeding. Of the patients whose ACTs were kept within target range, $10.6 \%$ had minor bleeding. It was the authors' opinion that once high-risk patients were identified, if ACTs were monitored closely and were kept below 190 seconds, major bleeding would not typically occur.

A final study that deserves mentioning is that of Conti and colleagues. ${ }^{20}$ Of the two groups compared, the first received what was termed highdose heparin; the 26 patients were administered a bolus of $150 \mathrm{U} / \mathrm{kg}$, which was followed by continuous intravenous infusions of 30 to $40 \mathrm{U} / \mathrm{kg} / \mathrm{hr}$. The second group, a conventional-dose group numbering 95 patients, received a bolus of 25 to $50 \mathrm{U} /$ $\mathrm{kg}$, with subsequent continuous intravenous doses adjusted to maintain the ACT within a therapeutic range of 150 to 190 seconds. These researchers reported prompt resolution of symptoms, including swelling and pain in a lower extremity, and no significant rethrombotic complications in the high-dose group; significant bleeding occurred in $8 \%$ of this population. In the conventional dose group, there were $10 \%$ thromboembolic complications, and bleeding occurred in $12 \%$.

Because of medical staff concern regarding highdose bleeding risk, the majority of patients on high doses were treated by the surgical department, while the medical department administered conventional-dose therapy to most of its patients. There was, therefore, a mixed population to some extent, without true randomization. The authors state that within the first 48 hours, rethrombosis and thrombus propagation of deep venous clots prove to be of greater risk than bleeding, provided that the patient does not have concomitant thrombocytopenia or other coagulopathic conditions.
They suggest that all patients at risk should be on the so-called high-dose regimen during the first 48 hours, and then continuous heparin maintenance should be adjusted according to the ACT.

\section{Present study}

The purpose of our study was to expand upon the research of Saltzman and associates ${ }^{16}$ and Wilson and coworkers. ${ }^{18}$ More specifically, we wished to answer the following questions: (1) Are there more complications, hemorrhagic $v$ rethrombotic, using continuous $v$ intermittent heparin? (2) If there are differences in complication rates, are they a matter of the amount of heparin administered, or associated with the "peaks and valleys" of anticoagulation, ${ }^{18}$ as would more likely be seen with intermittent bolus injection? (3) Is it necessary to monitor APTTs and adjust dosages accordingly?

\section{Methods}

The study had a two-factor design, in which patients were assigned randomly to one of four groups that either received intermittent or continuous intravenous heparin administration and that either did or did not include APTT adjustments. Twofactor analyses of variance and Mantel-Haenzel chisquare tests ${ }^{21}$ were used to determine whether there were significant differences among the factors and groups.

In group 1, patients received intermittent intravenous heparin without APTT adjustments, although the APTTs were monitored. This group received an initial 5,000 unit bolus (range, 50 to 125 $\mathrm{U} / \mathrm{kg}$, with an average of $71 \mathrm{U} / \mathrm{kg}$ and a mean of $75 \mathrm{U} / \mathrm{kg}$ ). Five-thousand units were administered intermittently every four hours thereafter. Group 2 patients received continuous intravenous heparin therapy without APTT adjustments, although these values were monitored. The initial bolus was 5,000 units (range, 60 to $90 \mathrm{U} / \mathrm{kg}$, with an average of only $67 \mathrm{U} / \mathrm{kg}$ and a mean of $68 \mathrm{U} / \mathrm{kg}$ ). Following the loading dose, the patient was given 1,250 units hourly.

The patients in group 3 received intermittent intravenous heparin, with APTT adjustments. A 5,000 -unit loading bolus (range, 48 to $100 \mathrm{U} / \mathrm{kg}$, with an average of $78 \mathrm{U} / \mathrm{kg}$ and a mean of $70 \mathrm{U} / \mathrm{kg}$ ) was followed by 5,000 units every four hours. Group 4 patients received continuous intravenous heparin, with an initial bolus of 5,000 units (range, 51 to $122 \mathrm{U} / \mathrm{kg}$, with an average of $65 \mathrm{U} / \mathrm{kg}$ and a mean of $59 \mathrm{U} / \mathrm{kg}$ ). This was followed by 1,250 units hourly, with adjustments according to APTTs.

Procedures. A complete copy of the study's proto- 
col was attached to the patient's chart. Informed and voluntary consent was obtained before any patient was treated. Following the intravenous bolus, for those on continuous infusion $30,000 \mathrm{U}$ of heparin $(1,000 \mathrm{U} / \mathrm{cc})$ were added to $570 \mathrm{cc}$ of dextrose in water. This was administered via an IMed pump at $25 \mathrm{cc} / \mathrm{hr}(1,250 \mathrm{U} / \mathrm{hr})$.

Contrast venography was performed for every patient for whom venous stasis was thought to be secondary to deep-vein thrombosis before the person entered the study, unless the condition was clinically obvious. Following entrance, if contrast venography was negative, the patient was removed from the study and another patient was assigned randomly.

Ventilation/perfusion (V/Q) lung scans were obtained initially when pulmonary embolus was suspected; they were repeated 10 days later to document improvement or when it was suspected that re-embolization had occurred during heparin therapy. In cases of recurrence, the patient was removed from the study, and the outcome was recorded.

Additional procedures were as follows: (1) Venography was not repeated unless pain and edema persisted; (2) every patient was required to undergo initial complete or, at least, portable chest roentgenography, which was to be repeated as needed; (3) initial arterial blood gas values were determined, with repeat tests as needed; (4) all stools and urines were Hemoccult $\mathrm{II}^{\circledR}$-tested daily; (5) APTTs were determined on a daily basis, either in the morning during continuous infusion, or 3 $1 / 2$ hours after the last intermittent administration of heparin; (6) a CBC, platelet count, and reticulocyte count was obtained every other day or as needed.

Criteria. Patients were considered to be at high risk if they met one or more of the following criteria: female sex, older than age 60; anatomic defect predisposing to bleeding (for example, peptic ulcer disease); surgery within previous 14 days; thrombocytopenia; uremia; intramuscular injections or intravenous cut downs; history of bleeding tendency; medication that interferes with platelet function; oral anticoagulant therapy; respirator hookup; severe hypertension (diastolic blood pressures $>115 \mathrm{~mm} / \mathrm{Hg}$ ); gastrointestinal bleeding within past six months; cerebrovascular accident within past 14 days.

Bleeding complications. Bleeding was defined as major or minor, as follows: Major bleeding included gross melena, gross hematuria, major hemorrhag- ing from surgical wounds, retroperitoneal bleeding or bleeding into closed spaces, bleeding into major organs, and bleeding necessitating transfusions.

Minor bleeding included occult blood in stools or urine, superficial ecchymoses, and wound hematomas.

Patient population. Thirty-three patients volunteered for the study and were considered evaluable. Nine were randomly assigned to group 1 , seven to group 2 , eight to group 3 , and nine to group 4 (Table 1). There were no significant mean differences among the groups $(F(3.29)=1.10)$. Ten of 17 patients $(59 \%)$ in groups 3 and 4 had proven pulmonary embolism, while $14(82 \%)$ had proven deepvein thrombosis. In groups 1 and 2,8 of 16 patients (50\%) had proven pulmonary embolism, while 12 $(75 \%)$ had proven deep-vein thrombosis. All pulmonary emboli were proven by a normal chest roentgenogram associated with a segmental V/Q mismatch demonstrated on high-resolution lung scanning, with the ratio improving later.

Ten of 17 patients $(59 \%)$ in groups 3 and 4 were considered to be at high risk for bleeding, while 10 of 16 patients $(63 \%)$ in group 1 and 2 were defined as such. Three of eight patients (38\%) in group $3 v$ eight of nine $(89 \%)$ in group 1 were at similar high risk. In group 4 , seven of nine $(78 \%)$ were at high risk, while only two of seven $(29 \%)$ in group 2 were considered as such.

\section{Results}

Bleeding. The incidence of bleeding in all four groups are delineated in Table 2. Four of nine patients (44\%) in group 1 bled; three were considered to have had major bleeding. Eight were considered to be high-risk patients. Gross hematuria occurred in a patient with known prostatic carcinoma that required an indwelling Foley catheter; he had had some microscopic hematuria prior to heparin therapy. Also, major bleeding occurred vaginally in a 34-year-old woman who had undergone total abdominal hysterectomy seven days earlier because of cervical carcinoma in situ. The third case of major bleeding involved a 48-year-old woman with known end-stage pancreatic carcinoma and metastases to liver and bones. She had multiple proximal femoral thrombi shown on the contrast venogram and an apical lung defect demonstrated on the V/Q lung scan. Major gastrointestinal bleeding ensued on day 8 of heparin therapy, after $\mathrm{He}$ moccult $I I^{\circledR}$-positive stools had been documented on day 6. Minor bleeding occurred in a 68-year-old 
TABLE 1. CHARACTERISTICS OF 33 PATIENTS TREATED WITH INTERMITTENT OR CONTINUOUS INTRAVENOUS HEPARIN.

Total number of patients

Age in years (mean $\pm \mathrm{SD})$

Sex

Male

Female

Primary diagnosis

Proved pulmonary embolism

Proved deep-vein thrombosis

Both pulmonary embolism and deep-vein embolism

Bleeding risk

High

Low
Standard dose based

on ideal body weight

Intermitten

(group 1)

9

$52.22 \pm 11.03$

3

8

1

\section{Continuous} (group 2)

7

$58.57 \pm 18.24$

\section{4}

3

4

2

2

5
Dose controlled

by APTT

$\overbrace{\text { Intermittent }}^{\text {Continuous }}$

$8 \quad 9$

$4 \quad 4$

$4-5$

$0 \quad 3$

$6-1$

25

327

$5-2$

woman with Dukes' stage D colon carcinoma and left upper lobe pneumonia with cavitation. She developed Hemoccult $\mathrm{II}^{\circledR}$-positive stools, which had required discontinuation of heparin therapy on day 3.

Of the seven patients in group 2, two were considered to be at high risk for bleeding. No complications developed in this group.

In group 3 , there were eight patients, with three in the high-risk category. One patient experienced minor bleeding. Because of her sex and age (85 years), she had been considered to be at high risk; also, she had undergone major surgery to resect a cecal carcinoma eight days earlier.

Seven of the nine patients in group 4 were considered to be at high risk to bleed. The high-risk patients experienced bleeding difficulties; one of them also had a concomitant recurrence of thrombosis. The first, a 95-year-old woman, had associated azotemia, with a serum creatinine value of $5.3 \mathrm{mg} / \mathrm{dL}$. She also had a history of hypertension, but her diastolic pressure readings were not excessively high. A V/Q lung scan of the second patient, an 85-year-old woman, had demonstrated a large mismatch in the right lower lung, and by day 7 of therapy, repeat $\mathrm{V} / \mathrm{Q}$ scanning demonstrated a large defect in the left apex as well as the original defect. Coffee-ground emesis appeared on days 6 and 7 , and bright red rectal bleeding was seen on

\begin{tabular}{|c|c|c|c|c|}
\hline \multirow[b]{2}{*}{ Bleeding } & \multicolumn{2}{|c|}{$\begin{array}{l}\text { Standard dose based } \\
\text { on ideal body weight }\end{array}$} & \multicolumn{2}{|c|}{$\begin{array}{c}\text { Dose controlled } \\
\text { by APTT }\end{array}$} \\
\hline & $\begin{array}{l}\text { Intermittent } \\
\text { (group 1) }\end{array}$ & $\begin{array}{l}\text { Continuous } \\
\text { (group 2) }\end{array}$ & $\begin{array}{c}\text { Intermittent } \\
\text { (group 3) }\end{array}$ & $\begin{array}{l}\text { Continuous } \\
\text { (group 4) }\end{array}$ \\
\hline Yes & 4 & 0 & 1 & 2 \\
\hline No & 5 & 7 & 7 & 7 \\
\hline
\end{tabular}

day 9 . The patient died during emergency colonoscopy.

There were no significant differences between any groups with respect to bleeding, according to the Mantel Haenzel chi-square test $\left(\mathrm{X}^{2}(1)=<.01\right)$.

Thrombosis. As shown in Table 3, recurrent thromboembolic problems occurred in two patients in group 1 and one patient in group 4 . In group 1 , a 67-year-old man developed superficial thrombophlebitis in his midthigh. The second group 1 patient, a 54-year-old man, had cardiac arrest and died. An autopsy was not granted, but it was the opinion of multiple observers that he had suffered massive pulmonary embolism. One group 4 patient, an 85-year-old female, considered at high risk by virtue of her sex and age, developed a rethrombosis. This was the same case as summarized above, whose complications were both bleeding and rethrombosis. Again, no significant differences in thrombosis rates were detected among the groups $\left(\mathrm{X}^{2}(1)=<.01\right)$.

\section{Discussion}

It was important to analyze the reported data in relation to associated prior risk factors. Group 1 had the highest number of bleeding incidences (4/ 9). All four patients had high-risk factors. In group 2 , there were no bleeding episodes, despite two of

\begin{tabular}{|c|c|c|c|c|}
\hline \multirow[b]{2}{*}{$\begin{array}{l}\text { Throm- } \\
\text { bosis }\end{array}$} & \multicolumn{2}{|c|}{$\begin{array}{l}\text { Standard dose based } \\
\text { on ideal body weight }\end{array}$} & \multicolumn{2}{|c|}{$\begin{array}{l}\text { Dose controlled } \\
\text { by APTT }\end{array}$} \\
\hline & $\begin{array}{l}\text { Intermittent } \\
\text { (group 1) }\end{array}$ & $\begin{array}{l}\text { Continuous } \\
\text { (group 2) }\end{array}$ & $\begin{array}{l}\text { Intermittent } \\
\text { (group 3) }\end{array}$ & $\begin{array}{l}\text { Continuous } \\
\text { (group 4) }\end{array}$ \\
\hline Yes & 2 & 0 & 0 & 1 \\
\hline No & 7 & 7 & 8 & 8 \\
\hline
\end{tabular}


seven patients being at high risk. Among the eight patients in group 3, there was one bleeding episode, in a patient who was one of three at highrisk. Seven of the 9 patients in group 4 were considered to be at high risk; two bleeding episodes were noted, with one patient concomitantly manifesting gastrointestinal hemorrhage and pulmonary rethrombosis.

A total of five bleeding episodes were seen in the intermittently dosed groups (groups 1 and 3), with all five patients at high risk. Four high-risk patients in group 1 and two high-risk patients in group 3 did not bleed. Neither of two high-risk patients in group 2 bled, nor did five of seven highrisk patients in group 4 . The APTTs for patients who did bleed were not significantly different from those who did not.

Somewhat unexpected in group 1 were the two thrombotic recurrences. Wilson and coworkers ${ }^{18}$ had described this type of problem mainly among patients given continuous infusion. They suggested the reason was simply that this population had received less heparin than those receiving intermittent bolus injections. they thought that the "peaks and valleys" theory of anticoagulation, as found with bolus injections, seemed less of a factor in rethrombosis. Both of our intermittent heparin patients with recurrent thrombosis were men and had never achieved therapeutic APTTs. One's APTT averaged only $1.3 \times$ baseline value, while his platelet counts progressively dropped after admission. His daily dosages of heparin averaged $352 \mathrm{U} / \mathrm{kg}$ $(14.7 \mathrm{U} / \mathrm{kg} / \mathrm{hr})$. Heparin-induced antiplatelet antibodies never were measured. The second patient's APTT averaged only $1.3 \times$ baseline, while his daily dose of heparin was lower at $238 \mathrm{U} / \mathrm{kg}(9.9 \mathrm{U} / \mathrm{kg} /$ $\mathrm{hr}$ ). The recurrent thrombotic incidence for the two intermittent heparin groups was $12 \%$

\section{Conclusions}

Because the group sizes were less than 20 patients per group, we consider the ability to generalize from study findings to be guarded. It is important to note, however, that although there was a trend for high-risk patients to fall into group 1 , the rates of bleeding and thrombotic incidences did not significantly differ across the groups. Although it is generally accepted that high-risk patients will be at greater risk for hemorrhage during heparin therapy, we did not find that the method of administration was a significant factor.
In our study, the APTTs did not appear to be a good predictor of hemorrhagic risk.

1. Hiebert LM, Jaques LB: The observation of heparin on endothelium after injection. Thromb Res 1976;8:195-204.

2. Mahadoo J, Hiebert LM, Jaques LB: Vascular sequestration of heparin. Thromb Res 1977;12:79-90.

3. Glimelius B, Buch C, Hook M: Binding of heparin on the surface of cultured human endothelial cells. Thromb Res 1978;12:773-782.

4. Estes JW, Poulin PF: Pharmacokinetics of heparin: Distribution and elimination. Thromb Diath Haemmorh 1975;33:26-37.

5. Nyman D, Thrunherr N, Duckert F: Heparin dosage in extracorporeal circulation and its neutralization. Thromb Diath Haemmorh 1975;33:102104.

6. Hirsch J, van Aken WG, Gallus AS, et al: Heparin kinetics in venous thrombosis and pulmonary embolism. Circulation 1976;53:691-695.

7. Simon TL, Hyers TM, Gaston JP, et al: Heparin pharmacokinetics: Increased requirements in pulmonary embolism. $\mathrm{Br} J$ Haematol 1978;39:111-120.

8. Thomas DP: Treatment of pulmonary embolic disease: A critical review of some aspects of current therapy. $N$ Engl J Med 1965;273:885892.

9. Chiu HM, van Aken WG, Hirsch J, et al: Increased heparin clearance in experimental pulmonary embolism. J Lab Clin Med 1977;90:204-215. 10. Teien AN: Heparin elimination in patients with liver cirrhosis. Thromb Haemost 1977;38:701-706.

11. Perry PJ, Herron, King JH: Heparin half-life in normal and impaired renal function. Clin Pharmacol Ther 1974;16:514-519.

12. Biggs R, Denson KW, Akman N, et al: Antithrombin III, antifactor $\mathrm{Xa}$ and heparin. Br J Haematol 1970;19:283-305.

13. Rosenberg RD: The chemistry of the hemostatic relationship mechanism and its relationship to the action of heparin. Fed Proc 1977;36:1018.

14. Cees AM, Nijmeyer B, Roelofs JM, et al: Kinetics of intravenously administered heparin in normal humans. Blood 1982;60:1251-1258.

15. Friedman Y, Arsenis C: Studies on the heparin sulfamidase activity from rat spleen: Intracellular distribution and characterization of the enzyme. Biochem J 1974;139:699-708.

16. Saltzman EW, Deykin D, Shapiro RM, et al: Management of heparin therapy: Controlled prospective trial. N Engl $J$ Med 1975;292:10461050 .

17. Glazier RL, Crowell EB: Randomized prospective trial of continuous vs intermittent heparin therapy. JAMA 1976;236:1365-1367.

18. Wilson JE, Bynum LJ, Parkey RW: Heparin therapy in venous thromboembolism. Am J Med 1981;70:808-816.

19. Hattersley PG, Mitsouka JC, King JH: Heparin therapy for thromboembolic disorders: A prospective evaluation of 134 cases monitored by the activated coagulation time. JAMA 1983;250:1413-1416.

20. Conti S, Daschback M, Blaisdell FW: A comparison of high-dose versus conventional-dose heparin therapy for deep vein thrombosis. Surgery 1982;92:972-978.

21. Fleiss JL: Statistical Methods for Rates and Proportions. New York, John Wiley and Sons, Inc, 1973.

From Kennedy Memorial Hospitals-University Medical Center and University of Medicine and Dentistry-School of Osteopathic Medicine, Stratford, NJ (Dr Freed, Section of Hematology/ Oncology), (Dr Morley, Respiratory Intensive Care Unit), (Dr Daly, Cardiac Catheterization Unit), (Dr Giudice, Division of Pulmonary Disease), and (Dr Steer, Department of Psychiatry).

Dr Freed, UMDNJ/SOM, Department of Internal Medicine, 301 S Central Plaza, Suite 3100, Stratford, NJ 08084-1504. 